\title{
Improved Algorithms for Data Visualization in Forensic DNA Analysis
}

\author{
Noor Maizura Mohamad Noor, Senior Member IACSIT, Mohd Iqbal Hakim Harun, and Ahmad Faiz \\ Ghazali
}

\begin{abstract}
DNA profiles from forensic evidence have contributed significantly in criminal investigations. DNA profiles can be developed using several techniques including Short Tandem Repeat (STR) analysis. The algorithm for data visualization in forensic DNA analysis will be employed into a series of formulas by using a mathematical model that will be represented by a graphical model. By applying visualization, it can increase the value of using linear planning charts which, when combined with images pertaining to the distribution of resource usage in time and space, permits additional insights on the quality of a schedule to be glean.
\end{abstract}

Index Terms-Data visualization, DNA, statistical analysis.

\section{INTRODUCTION}

Malaysia has been experiencing accelerated urbanization since the last two decades and the crime rate in Malaysia is now reaching sky-high, generating considerable fear within the community [1], making safety an issue which demands critical attention at both the local and national level. Similarly, the rising unemployment rate is caused by the economic cycle. In the United Kingdom, they estimated that crime can cost more than 60 billion pounds per year, which is more than $5 \%$ of the national income [2].

In the Malaysian context, there are several street crimes which include three types of crime index, thefts, robberies without firearms and gang robbery without firearms [2]. The crime rate is high and constantly rising, which in turn will give an impact on economic growth, particularly overseas view on safety in our country.

There are also other categories of crime index. These crimes include incidents of substance abuse, white collar crime (commercial crime), and illegal gambling syndicate [2].

The government has taken steps in reducing and tries to ultimately overcome these criminal cases by using new modern technology including science and information technology.

Science has been the main field to the modern world

Manuscript received June 11, 2013; revised October 4, 2013. Improved Algorithms for Data Visualization in Forensic DNA Analysis. This work was supported in part by Fundamental Research Grant Scheme (FRGS) from the Ministry Of Higher Education (MOHE) using vot number 59289 and Exploratory Research Grants Scheme (ERGS) from the Ministry Of Higher Education (MOHE) using vot number 55077 and eScienceFund from the Ministry of Science, Technology and Innovation (MOSTI) using vot number 52056

Noor Maizura Mohamad Noor, Mohd Iqbal Hakim Bin Harun and Ahmad Faiz Ghazali are with University Malaysia Terengganu, 21300 University Malaysia Terengganu (UMT),Terengganu, Malaysia (email:maizura@umt.edu.my,maizuraumt@gmail.com). where many invention and discovery were introduced to the modern society and in modern science, there are many fields that lead to the prosperity of human life. There are a few examples in DS that are being used worldwide. For example, it is often mentioned in connection with Data Warehouses and others are to associate DS with Data Mining. Nowadays, both of these examples are combined in order to support difficult real-life problem solving and more importantly DS can give many contributions to solve crime cases [3].

One of the fields in DS is the finding of the functionality of genetic in deoxyribonucleic acid (DNA). Although the term "Decision Support" seems rather intuitive and simple, it is in fact very loosely defined. It means different things to different people and in different contexts.

Through the detailed observation on how the whole system operates, an extensive study has been made on how to improve the current methodology for the multidisciplinary research contributions in Computer Science and Forensic Science. It is crucial to do research on what other researchers are exploring for data visualization in the fields of Computer Science and Forensic Science.

\section{VisUALIZATION OF DNA ANALYSIS FOR CRIMINAL INVESTIGATION}

DNA evidence is among the evidence that arise in court, in offering scope for mathematical treatment. In this project, we shall develop improved algorithms for processing data for DNA analysis particularly in data visualization for forensic DNA analysis in crime investigations.

In this research we will develop improved algorithms using data visualization for forensic DNA analysis in crime investigation. Different criteria are proposed to search algorithms, based on choosing a parameter configuration that optimizes the performance of the algorithm in order to minimize the overall expected runtime [3]. All that is required for the model selection approach to be applicable to a search algorithm is a problem instance and parameters. The study on the existing analytical techniques used for DNA profiles includes CODIS (Combined DNA Index System) from United States, NDNAD (National DNA Database) from United Kingdom and FDDM (Forensic DNA Database Malaysia) from Malaysia. The general goal of statistical approach (or statistical estimation theory) [4] is to estimate some unknown parameter (of algorithms) from the observation of variables or data

Statistical aproach in DNA evidence has been utilized widely in cases of forensic identification over the last few years [5] until this year [6], [7]. The strength of this 
evidence against another trial has been used to be presented in court in the form of LR [7]. The ideas and methods for analyzing data and evaluating LRs where Bayesian approach is given special attention [8], [9] and when the evidence is based on STR (short tandem repeat) profiles, data visualization is among the most important part in representation of data for analysis and summary of results.

There are many type of visualization that can be used in conveying data, for example the use of graph ordering algorithms for visual analysis of data sets using visual similarity matrices are studied by. The biggest challenge for displaying data using this representation is finding an ordering of the data items that reveals the internal structure of the data set," Researchers considered three general classes of algorithms for generating orderings:

1) Simple graph theoretic algorithms

2) Symbolic sparse matrix reordering

3) algorithms.

4) Spectral decomposition algorithms

The evaluated algorithm then was revised and extended to a version of the top for the unsolved problems of information visualization has been outlined in IEEE Visualization 2004 panel. The problems are not limited to technical issues, but more into the growth of information visualization as a field on different levels and perspectives

In the DNA analysis, the DNA technology has proven to be very useful for criminal identification, but the country's policies are strict which requires a standard procedure to analyze and evaluate DNA evidence.

\section{Problem Statement}

Constraints of current Bayesian methodology employed in forensic DNA analysis is that it does not consist of learning due to one of the fix world standard formula employed for forensic DNA analysis for STR. Hence, additional suggestion is to add data visualization concept to firm, specifically utilizing concept information visualization (IV). Lack of proper visualization techniques in analyzing data in one shot might slow down crime investigation and the worst part is, the case would remain unsolved. The main objective is to design the improved algorithms using data visualization for forensic DNA analysis in a new architecture to handle DNA profiles for identification of possible criminals and convicted offenders [10]. The second objective from this research is to implement the improved algorithms of information visualization to analyze DNA profiles based on patterns and relations. Lastly the main objective is to evaluate the proposed algorithms using data visualization for forensic DNA analysis and statistical analysis in crime investigation.

With these objectives, it motivated us to get involved in this project because research for computerized DNA analysis is getting more attention due to its importance in scientific discovery, as well as for forensic DNA analysis in determining family traits, natural disaster recovery and crime investigation [10]. The reason why we chose data visualization techniques to be employed in forensic DNA analysis is because of the constraint of current Bayesian methodology, specifically the mathematical model to get the random match probability and allele frequencies for STR.
According to senior forensic scientists, the world's standard mathematical formula employed still has not changed and can only be changed by experts in Forensic Science.

Similar limitations of the effectiveness of representing results effectively to the user were found by multidisciplinary research in recent years using the few suggested visualization techniques. Data visualization can assist with at least three significant needs, where firstly it can assist with analytical reasoning, secondly in communication, and lastly it will assist in learning of how to convert the data to suit certain cases [11].

One of the most significant result of applying visualization as explained. On others' work is visualization can increase "the value of using linear planning charts which, when combined with images pertaining to the distribution of resource usage in time and space, permitted additional insights on the quality of a schedule to be gleaned," Hence, to seek a structured way of thinking that will help formulate visual images of data and supporting interaction features.

\section{Methodology}

Forensic mathematics concept are also being applied in the form of likelihood ratio (LR) or DNA profile match probability for such cases. Research in forensic mathematics and statistical analysis have been explored by several researchers [11], [12]. In these kinds of cases, it is natural to construct the Bayes' linear graphical model by writing each random quantity in the model as a node on the diagram. Likelihood ratio (LR) shows the forensic impact of DNA evidence by comparison of hypotheses [13].

This is because LR is one of the famous methods used to generate multiple mathematical models in order to provide explanations for DNA data analysis [14], [15]. LR and its correspondence weight is then be used as a basis to determine the strength of DNA evidence against the suspect [16].

To solve the crime cases, the algorithms used for automatic parameter in Bayesian networks are being demonstrated. Bayesian networks (BNs) are an established framework in order to handle uncertainty in artificial intelligence [17], [18]. Bayesian approach presented is able to supply mechanism for learning parameters. Concept of probabilistic inference algorithms can be easily understood using the method proposed known as singly connected Bayesian networks (SCBNs) that is a special case of BNs. Concept of probabilistic inference is being used in relation with Bayesian networks by [19].

Users spend a lot of time tuning algorithms for their specific problem domain, and this time could be saved by an automatic approach for parameter tuning. Researchers [19] define the semantics of Bayesian networks in a form of joint distribution. A novel method where it is constructed from the probability distributions of DNA sequence. The directed acyclic graph is a $\mathrm{BN}$ relative to a set of variables if the joint probability distribution of the node variables can be written as the product of the local distributions of each node and its parents. Bayesian networks approach like this is employed by [19] to a set of variables of each node as: 


$$
\mathrm{P}\left(x_{1} \ldots x_{n}\right)=\prod_{i}^{n} P\left(x_{i} \mid \operatorname{parents}\left(x_{i}\right)\right)
$$

Referring mathematical model above, they classify instances $i$ given by certain characteristics $x_{i}=\left(x_{i}, \ldots, x_{i n}\right)$ into r class labels, $c_{i}, i=1, \ldots$, r. $x_{i l}$ denotes the value of variable $x_{1}$ as observed in instance $i$. The main principle of a Bayesian classifier is the application of Bayes' theorem. Using Bayes' theorem, probabilities can be calculated using mathematical models.

Researcher [20] described the formula for likelihood ratio as follows. Since prosecution's hypothesis is that the suspect committed the crime, they simplified it with $\mathrm{H}_{0}=1$. Researchers [20] demonstrated the general idea of this formula as below;

$$
L R=\frac{\mathrm{P}\left(\mathrm{E} \mid \mathrm{H}_{0} \mathrm{I}\right)}{P\left(E \mid \mathrm{H}_{1 . \mathrm{I}} \mathrm{I}\right.}=\frac{1}{P\left(E \mid H_{1 . \mathrm{I}}\right)}
$$

\section{$H_{0}=$ The source of DNA is from the suspect$$
H_{1}=\text { The source of DNA is from others }
$$

\section{Method \#1}

Assuming suspect is not related to the crime. Calculation for genotype frequency [20]

$$
P\left(E \mid H_{1}, I\right)=\left\{\begin{array}{c}
p_{i}^{2}, G c=G s=A_{i} A_{i} \\
2 p_{i} p_{j}, G c=G s=A_{i} A_{j}
\end{array}\right.
$$

\section{Method \#2}

To allow relatedness of the crime with suspect's subpopulation. Calculation for genotype frequency allowing population structure [20];

$$
\begin{aligned}
& \frac{\left[2 \theta+(1-\theta)_{p i}\right]\left[3 \theta+(1-\theta)_{p i}\right]}{(1+\theta)(1+2 \theta)}, G_{c}=G_{s}=A_{i} A_{i} \\
& P\left(\mathrm{E} \mid \mathrm{H}_{1}, \mathrm{I}\right)=\frac{\left.2 \theta+(1-\theta)_{p}\right]\left[3 \theta+(1-\theta)_{p j}\right]}{(1+\theta)(1+2 \theta)}, G_{c}=G_{s}=A_{i} A_{j}
\end{aligned}
$$

A mathematical model through Bayesian networks using an extended LR approach:

$$
L R=\frac{\operatorname{Pr}\left(E \mid H_{p}\right)}{\operatorname{Pr}\left(E \mid H_{d}\right)}=\frac{\operatorname{Pr}\left(E \mid H_{p}\right)}{\sum_{i=2}^{N} \operatorname{Pr}\left(E \mid H_{i}, H_{d}\right) \operatorname{Pr}\left(H_{i} \mid H_{d}\right)}
$$

where;

\section{$H_{p}:$ The DNA came from the suspect $H_{d}$ :The DNA came from a male not related to the suspect}

The formula above is proposed after reviewing some current methods including LR and its limitations. A brief idea of a domain with variables of interest: $\mathrm{X}=\left\{X_{1}, \ldots, X_{n}\right\}$ where a $\mathrm{BN} B$ is a probabilistic model that encodes the joint distribution of domain variables. The nodes in a BN can be utilized to represent variables of interests.

Realizing the significant of applying mathematical formula in crime investigation, [21] mentioned about crime and concentrated more about database rate for match rate calculation. Researchers [22] explained Bayesian fusion that also utilized probabilistic concept with a clearer idea plus its relation with criminal investigation [22]. Another method of calculation for likelihood ratio like other researchers and claims to provide a better measurement of the strength of evidence because it considers the presence of rare alleles. This will increase the probability of detection because it allows a large number of relatives to be linked by likelihood ratio.

The papers by [22] present two rectangle-packing algorithms, means to place items of hierarchical data onto display spaces. Researcher [22] presents "a technique for the representation of large-scale hierarchical data which aims to provide good overviews of complete structures and the content of the data in one display space. The technique represents the data by using nested rectangles," Researchers [23] present I-PACK, a deterministic layout algorithm for embedding a data set $\mathrm{X}$ in $2 \mathrm{D}$ provided that distances between data items are given or can be computed. They performed experimental comparisons with other multidimensional scaling algorithms.

Many researchers work on extending kernel methods to interval data mining and using graphical methods to explain the obtained results, where no algorithmic changes are required.

From the entire algorithm that being used we proposed a new architecture for this DNA analysis and visualization

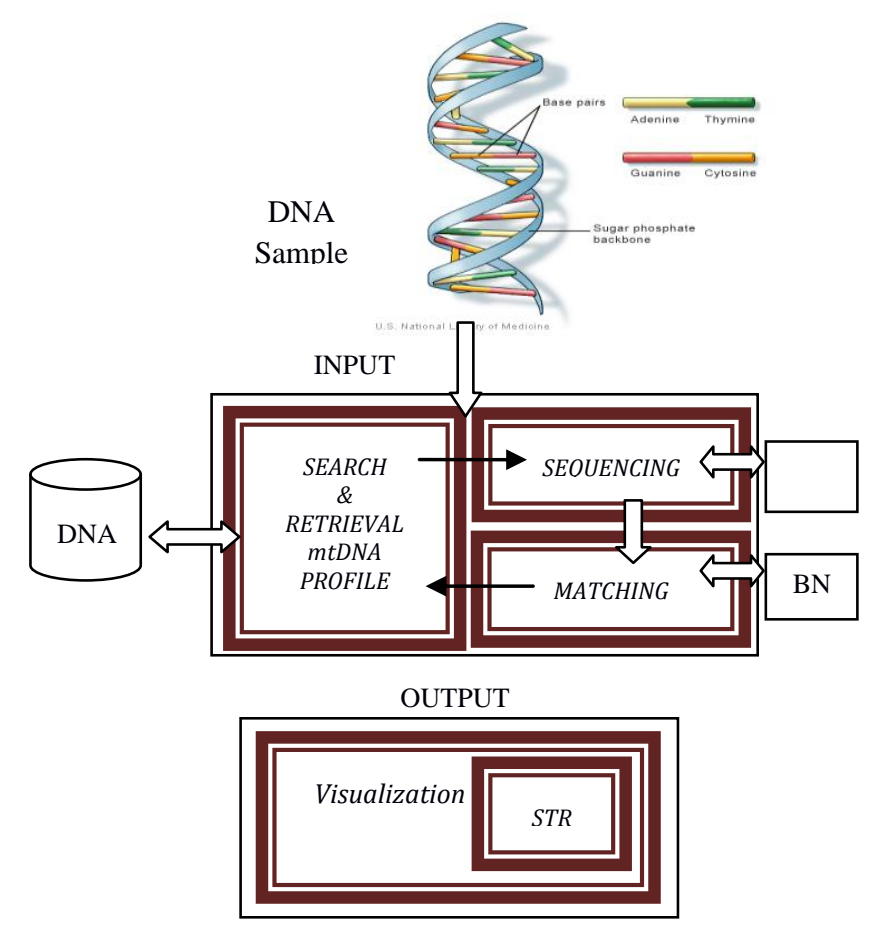

Fig. 1. Forensic DNA Analysis visualization architecture.

\section{RESEARCH PROPOSED}

For the visualization in the DNA analysis we propose several phases to analyze the data. For phase 1, we will 
design the improved algorithms using data visualization for forensic DNA analysis to handle the DNA profiles for identification of possible criminals and convicted offenders. Then for Phase 2, we will implement improved algorithms using data visualization for forensic DNA analysis to analyze DNA profiles based on patterns and relations. Designs for algorithms will be based on elements that have been defined in Phase 1.

\section{EXPECTED BENEFIT}

This study can bring benefits in data visualization and scientific visualization as well. Researchers [9] listed ten guidelines for effective data visualization in scientific publications, which claimed they support the main objective of data visualization; to effectively convey information.

Interactions between visual representations allow users (where in this context are referring to scientists) to have one overall view of complex data presented. This will be able to help scientists to gain important insights by interpreting the data for analysis according to their expertise through images presented.

The concept of visual analytics can be applied. Interactions between visual representations allow users (in this context scientist) to have one overall view of complex data presented. It is expected to be understood well.

Based on extensive study on literature reviews, the best proposed work can be developed and as general suggestions specifically for scientific visualization across a range of disciplines where data visualization has been demonstrated in many places that it can "improve decision-making and provide a better reflection of our scientific understanding" based on work by many researchers in scientific fields reviewed by them.

\section{CONCLUSION}

Performance analysis and technology transfer of algorithms will be evaluated in terms of its performance. Produced work will be evaluated by domain experts in Computer Science and Forensic Science as well including forensic scientists in terms of its usability and contribution towards utilizing computer science and information technology for crime investigation. The methodology for this research uses the concept of Bayesian.

\section{ACKNOWLEDGEMENT}

The author would like to thank Datuk Seri Mohd Bakri Bin Mohd Zini, Deputy Inspector General of Police (IGP), and Royal Police Malaysia (RPM) for his continuous support. This work was supported by research grant from Exploratory Research Grant Scheme (ERGS), Ministry of Higher Education (MOHE) Malaysia, with two vot number 55077 and 59289, eScienceFund Ministry of Science, Technology and Innovation (MOSTI) with vot number 52056.

\section{REFERENCES}

[1] M. A. M. H. M. H Elsawahli, "Crime And Housing In Malaysia: Case Study Of Taman Melati Terrace Housing In Kuala Lumpur,"
Asian Journal of Environment-Behaviour Studies, no. 3, September 2010

[2] A. A. S. M. M. F. Hashim, "Kesan Kitaran Ekonomi Terhadap Peningkatan Kadar Indeks Jenayah di Malaysia," Prosiding perkem, vol. VI. 2011.

[3] R. Pavón et al., "Automatic parameter tuning with a Bayesian casebased reasoning system," A case of study. Expert Systems with Applications, vol. 36, no. 2, Part 2, pp. 3407-3420, 2009.

[4] R. Pavón, F. Díaz, and V. Luzón, "A model for parameter setting based on Bayesian networks," Engineering Applications of Artificial Intelligence, vol. 21, no. 1, pp. 14-25, 2008.

[5] L. A. Foreman, A. F. M. Smith, and I. W. Evett, "Bayesian Analysis of DNA Profiling Data in Forensic Identification Applications," Journal of the Royal Statistical Society: Series A (Statistics in Society), vol. 160, no. 3, pp. 429-459. 2007.

[6] P. Larrañaga and S. Moral, "Probabilistic graphical models in artificial intelligence," Applied Soft Computing, vol. 11, no. 2, pp. 1511-1528, 2011.

[7] C. Yu, M. Deng, and S. S. T. Yau, "DNA sequence comparison by a novel probabilistic method," Information Sciences, vol. 181, no. 8. 2011.

[8] M. Munetomo, N. Murao, and K. Akama, "Introducing assignment functions to Bayesian optimization algorithms," Information Sciences, vol. 178 , no. 1, pp. 152-163, 2008

[9] M. Correa, C. Bielza, and J. Pamies-Teixeira, "Comparison of Bayesian networks and artificial neural networks for quality detection in a machining process," Expert Systems with Applications, vol. 36, no. 3, Part 2, pp. 7270-7279, 2009.

[10] C. H. Brenner, "Fundamental problem of forensic mathematics--The evidential value of a rare haplotype," Forensic Science International: Genetics, vol. 4, no. 5, pp. 281-291. 2010.

[11] M. Goldstein and D. Woof, "Bayes Linear Statistics: Theory and Methods," in Wiley Series in Probability and Statistics, P. B. David, J. Balding, and N. A. C. Cressie, ed., Durham University, UK: Wiley, 2007.

[12] P. Gill, J. Curran, and C. Neumann, "Interpretation of complex DNA profiles using Tippett plots," Forensic Science International: Genetics Supplement Series, vol. 1, no. 1 pp. 646-648, 2008.

[13] J. S. Buckleton, C. M. Triggs, and C. Champod, "An extended likelihood ratio framework for interpreting evidence," Science \& Justice, vol. 46, no. 2, pp. 69-78, 2006.

[14] S. Cowen and J. Thomson, "A likelihood ratio approach to familial searching of large DNA databases," Forensic Science International: Genetics Supplement Series, vol. 1, no. 1, pp. 643-645, 2008.

[15] J. Drábek, "Validation of software for calculating the likelihood ratio for parentage and kinship," Forensic Science International: Genetics, vol. 3, no. 2, pp. 112-118, 2009.

[16] A. Roy and R. Leiva, "Likelihood ratio tests for triply multivariate data with structured correlation on spatial repeated measurements," Statistics \& Probability Letters, vol. 78, no. 13, pp. 1971-1980, 2008.

[17] C. J. Butz et al., "A simple graphical approach for understanding probabilistic inference in Bayesian networks," Information Sciences, vol. 179, no. 6, pp. 699-716, 2009.

[18] B. R. Cobb and P. P. Shenoy, "Inference in hybrid Bayesian networks with mixtures of truncated exponentials," International Journal of Approximate Reasoning, vol. 41, no. 3, pp. 257-286, 2006.

[19] M. Chavira and A. Darwiche, "On probabilistic inference by weighted model counting," Artificial Intelligence, vol. 172, no. 6-7, pp. 772-799, 2008.

[20] N. M. M. Noor and R. Baital, "An Integrated DNA Support System for Crime Investigations: DNA Tracers," Journal of Ultra Scientist of Physical Sciences, vol. 21, no. 3, pp. 989-998, 2009.

[21] J. Buckleton, J.-A. Bright, and S. J. Walsh, "Database crime to crime match rate calculation," Forensic Science International: Genetics, vol. 3, no. 3, pp. 200-201, 2009.

[22] J. Sander and J. Beyerer, "A local approach for Bayesian fusion: Mathematical analysis and agent based conception," Robotics and Autonomous Systems, vol. 57, no. 3, pp. 259-267, 2009.

[23] A. Don and N. Hanusse, "A Deterministic Multidimensional Scaling Algorithm for Data Visualization," Tenth International Conference on Information Visualization (IV'06), vol. iv, pp. 511-520, 2006.

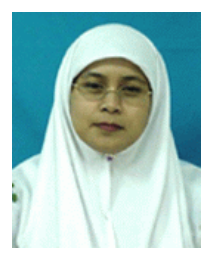

Noor Maizura Mohamad Noor is an Associate Profesor at Faculty of Science and Technology, Universiti Malaysia Terengganu (UMT). She received her PhD degree from The Manchester University, UK in 2005. Her recent research work focuses on improving organizational decision making practices through the use of technologies. She is an editor of books, conferences proceedings and also a reviewer 
and editorial board of International Journals. Her contribution to scientific computing has been recognized by receiving several awards including the Best Scientist Award (2009), Product and Research Innovation Award for International and National Level (2008 and 2012) of UMT. She is a senior member IACSIT (80337401), member of ACM and IACSIT.

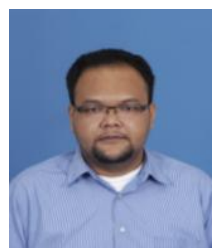

Mohd Iqbal Hakim Bin Harun is currently a master degree student in Computer Science Department, University Malaysia Terengganu. He received first degree in Computer Science majoring in Software Enggineering from University Malaysia Terengganu in 2012. His researchs interest are information system, decision support system.

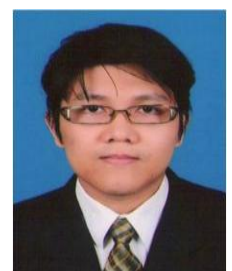

Ahmad Faiz Ghazali obtained his bachelor degree in computer science from Faculty of Computer Science, Universiti Sains Malaysia in 2008. He is now a postgraduate student at Computer Science Department, Faculty of Science and Technology, Universiti Malaysia Terengganu, Malaysia. His research interests are software engineering and decision support system. 